\title{
Caracterización de la
} primera generación de graduados en diseño de producto 2013-2017 Análisis de proyectos finales registrados en las Medallas ADI FAD

\author{
Guillem Ferran Masip; Universitat Ramon Llull. guillemfm2@blanquerna.url.edu
}

recepción: 04-06-2018, aceptación: 05-09-2018, publicación: 15-01-2019

\section{Resumen}

Esta investigación analiza todos los trabajos inscritos en el concurso Premios Delta Medalla ADI de las ediciones del 2014, 2016 y 2018. Nos hemos centrado en el análisis de contenidos siguiendo una serie de indicadores. Los proyectos presentados: los temas y los ámbitos de los proyectos finales, y de manera complementaria de los perfiles de los participantes. Los datos se han recogido siguiendo una serie de indicadores que pueden aportar información de los proyectos finales que se están realizando en los diferentes estudios de enseñanza, relacionados con el diseño y sobre todo con el diseño industrial o diseño de producto durante las promociones comprendidas entre los años 2013 y 2017.

El trabajo consiste en la compilación de los datos de los participantes y sus trabajos, para elaborar un mapeo de la primera generación de estudiantes de los títulos oficiales de diseño. Al mismo tiempo esta muestra es una exposición de la tipología de trabajos finales de curso que se realizan en las escuelas de diseño de producto de España.

\section{Palabras clave}

Educación superior diseño; concursos de estudiantes de diseño de producto; escuelas de diseño; proyectos finales de estudio; generación 2013-2017

Characterization of the first generation of graduates in product design 2013-2017.

Analysis of final projects registered in the ADI FAD Medals.

Abstract

This research analyzes all the works registered in the ADI Medal of de Delta Awards from ADIFAD contest of the editions of 2014, 2016 and 2018. We have focused on content analysis following a series of indicators. The projects presented: the themes and areas of the final projects, and in a complementary way to the profiles of the participants. The data has been collected following a series of indicators that can provide information on the final projects that are being carried out in the different education studies, related to design and especially to industrial design or product design during promotions included in the 2013 and 2017

The work consists in the compilation of the data of the participants and their works, to elaborate a mapping of the first generation of students of the official titles of design. At the same time, this exhibition is an exhibition of the typology of final course projects that are carried out in the schools of product design in Spain.

Keywords

Higher education; student competitions of product design; design schools; final projects of study; generation 2013-2017 


\section{Introducción}

El trabajo se centra en un análisis de contenidos con la voluntad y finalidad de recoger datos que puedan ser útiles como representación de los trabajos de finales de estudios que están realizando los estudiantes de las diferentes escuelas de diseño de producto que participan en el concurso.

El objetivo del estudio es realizar un mapa de datos sobre las tendencias de diseño de producto y las tipologías de proyectos de todos los alumnos ya graduados y los perfiles de los participantes en el concurso con sus proyectos de fin de estudios.

Este estudio permite también ser una guía para las siguientes generaciones al identificar tendencias en áreas de interés, con referencia a los futuros proyectos finales de estudios sobre las temáticas, tipos de proyectos.

Los estudios oficiales en diseño empezaron a impartirse en 2009 en la mayoría de centros. Esta investigación pretende ser una muestra de la evolución de los proyectos finales de diseño de producto desde la implementación de los actuales planes de estudio de la primera generación, que comprende las cinco primeras promociones que finalizaron desde 2013 a 2017.

Las Medallas ADI son unos premios con una gran tradición en el panorama de diseño industrial de España para estudiantes de diseño industrial. Creadas el año 1976 como una convocatoria en paralelo a los Premios Delta del ADI-FAD. Los Premios Delta de ADI-FAD, de la Asociación de Diseño Industrial del Fomento de las Artes y el Diseño, se otorgan desde 1961 en reconocimiento al trabajo de los diseñadores y de las empresas nacionales e internacionales, para celebrar la excelencia en el diseño industrial y reconocer su importancia como expresión de los valores económicos, sociales y culturales.

Las Medallas ADI son un concurso de inscripción gratuita que puede realizarse por iniciativa propia, por invitación o por sugerencia de los observadores, que son personas buscadas por el ADIFAD en la mayoría de las escuelas de Diseño de España. Con el tema abierto, la convocatoria se dirige a jóvenes diseñadores recién graduados y tiene como objetivo contribuir a establecer puentes entre el ámbito académico y el mundo industrial y comercial, realizando una muestra de los mejores Proyectos Finales que se realizan en el estado Español.

\section{Desarrollo}

El trabajo analiza los proyectos inscritos al concurso Premios Delta en las ediciones 2014, 2016 y 2018, en concreto en la Medalla ADI 2014, 2016 y 2018.

En los trabajos de fin de estudios los estudiantes deben buscar y encontrar el problema, la necesidad o la oportunidad en la que se identifican y creen que es estratégicamente importante y en la mayoría de casos le dan el enfoque hacia donde quieren desarrollar su profesión y el tipo de diseño o ámbito en el cual le gustaría enfocar su futuro.

Los concursos y medallas ADI-FAD son una buena motivación práctica para el alumnado. Permiten contrastar saberes académicos, aptitudes y competencias al enfrentarse con la realidad y dan a conocer los proyectos y trabajos de fin de carrera de los futuros diseñadores más allá de las escuelas.

El hecho de la participación en concursos externos al centro educativo y el ser juzgados o valorados por personal distinto de los profesores habituales, permite al propio estudiante unos grados de aprendizaje experiencial y vivencial, puesto que los criterios de evaluación del profesorado en los centros educativos, puede ser distinto de las propias bases u objetivos del concurso. Tomando en consideración que el proyecto final de estudios es el último proyecto que el alumno realiza en la escuela antes de su inmersión en el período profesional, el hecho de poder conseguir una valoración distinta le puede ayudar a establecer unos niveles de auto reflexión facilitadores de un aprendizaje competencial, que según Van Manen (1977): una reflexión técnica centrada en la eficiencia y eficacia sobre los medios utilizados para alcanzar el fin, una reflexión práctica sobre los objetivos y los resultados, adquiriendo en este tipo de reflexión una relevancia importante el significado en el lenguaje y la comunicación y una reflexión crítica que incorpora los dos niveles anteriores y que favorece el feed-back personal para una mejora y aprendizaje posterior experiencial y profesionalizado.

\section{Metodología}

Para llevar a cabo el estudio se han utilizado como fuentes los materiales del archivo ubicados en la base de datos del ADIFAD en Barcelona y también de la plataforma de registro a los Premios que utiliza el ADI FAD, conocida como "Typeform".

Los contenidos se han recogido de forma cualitativa, y cuantitativa para conectar i fusionar los dos tipos, con la finalidad de poder extraer algunas conclusiones más significativas.

Se ha llevado a cabo en tres fases ya que se han analizado con los mismos indicadores y variables elegidas, los tres periodos correspondien- 
tes a las diferentes convocatorias de los premios Delta 2014, 2016, y 2018.

El total de la muestra analizada es de 208 inscripciones a las Medallas ADI. Son 49 en 2014, 108 en 2016 y 51 en 2018. Se ha mantenido la confidencialidad de los inscritos y sus datos de carácter personal. Posteriormente se ha llevado a cabo una extracción de diferentes variables de tipo exploratorio e histórico según unos indicadores para comprobar, ilustrar o construir una teoría de los perfiles de participantes y de los tipos de proyectos y de su comunicación (Xavier Coller, 2000).

Se ha realizado el estudio a partir de dos unidades temáticas, la de los participantes y los proyectos realizados.

Los indicadores elegidos con referencia a los participantes han sido: El género, la presentación individual o en grupo del proyecto, la escuela donde ha estudiado, los estudios considerando MECES si es de $1^{\circ}$ nivel (CFGS), $2^{\circ}$ Nivel (Grado), $3^{\circ}$ Nivel (Máster) y la nacionalidad.

- Los indicadores de estudio desde la unidad temática de los proyectos presentados han sido:

- El Título. Teniendo en cuenta el idioma, el nombre del proyecto, si ha sido una palabra inventada.

- El ámbito del PFE. Caracterizado según las siguientes variables: producto hábitat, mobiliario, iluminación, tecnología, medicina, movilidad, juguetes, urbano...

- El tipo de proyecto: comercial, especulativo, experimental, social o servicio.

Los indicadores elegidos con referencia a los materiales complementarios han sido:

- Página Web. Si han aportado un enlace de Web personal.

- Materiales audiovisuales. Aquellos que han utilizado recursos complementarios u opcionales como son el vídeo o una página Web del Proyecto.

La relevancia de estos indicadores puede aportarnos si existen centros que incentivan más la participación que otros, si los participantes son todos de unos determinados grados o estudios o aparecen con mayor influencia en unos que en otros, si hay variabilidad significativa entre sexos. También pueden ayudarnos a identificar los temas y los ámbitos en los que se desarrollan los proyectos de final de carrera en los diferentes centros y a lo mejor se puede incluso augurar si con el paso del tiempo se mantienen los mismos o van variando y que elementos, innovaciones o influencias sociales o educativas van provocando los cambios en el caso de que los hubiera.

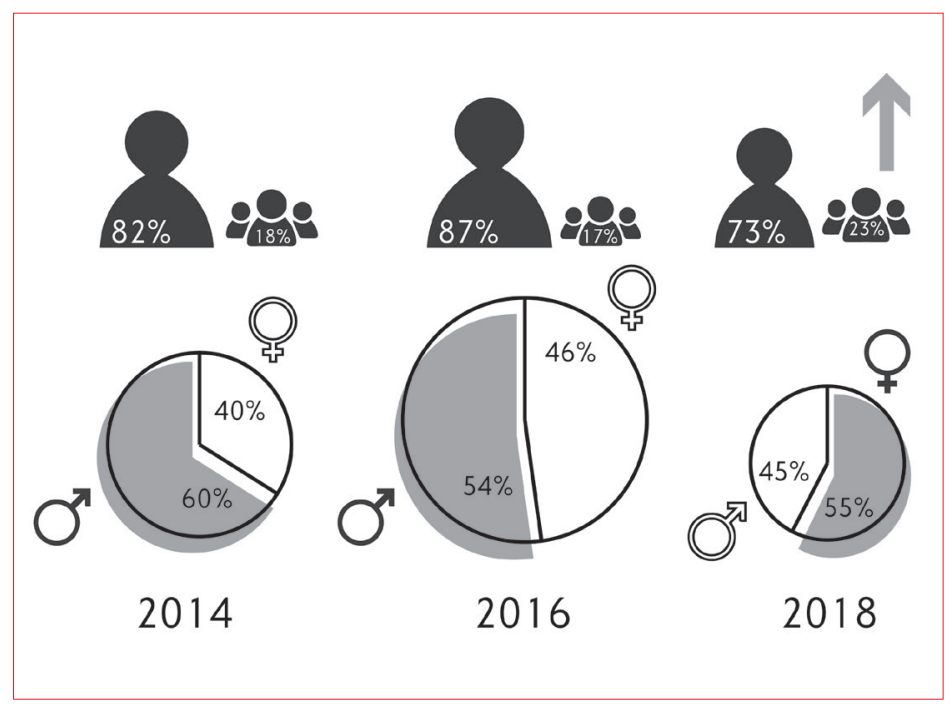

Figura 1 Perfiles de los participantes

\section{Resultados}

Una vez recogidos todos los datos cuantitativos y cualitativos, analizados los mismos y tomando un enfoque descriptivo-interpretativo de la actualidad que nos muestran, podemos disponer de un mayor conocimiento comprensivo de esta realidad compleja que además cuenta con pocos estudios transversales tanto de centros, como de estudios y proyectos.

No podemos exponer todos los resultados de todas las variables recogidas por la limitación del artículo, pero intentaremos exponer algunos de los más significativos y que permiten aportar reflexión al respeto.

\section{Perfiles de los participantes}

En el análisis de si los participantes han elegido una presentación de tipo individual o de trabajo en equipo podemos observar que mayoritariamente la tendencia es la presentación a título individual, como se muestra en la figura 1. Podemos entender esta posición desde el punto de vista de premio o concurso, pero hay una ligera tendencia al aumento de los trabajos finales realizados en equipo en los últimos años. Esta observación pone de relieve el trabajo cooperativo que en los últimos años se está llevando a cabo en muchos centros educativos.

Aprender a realizar proyectos con esta metodología de trabajo cooperativo enriquece y desarrolla el aprendizaje de habilidades y competencias transversales y generales que son necesarias en la formación de los diseñadores. En un futuro tam- 


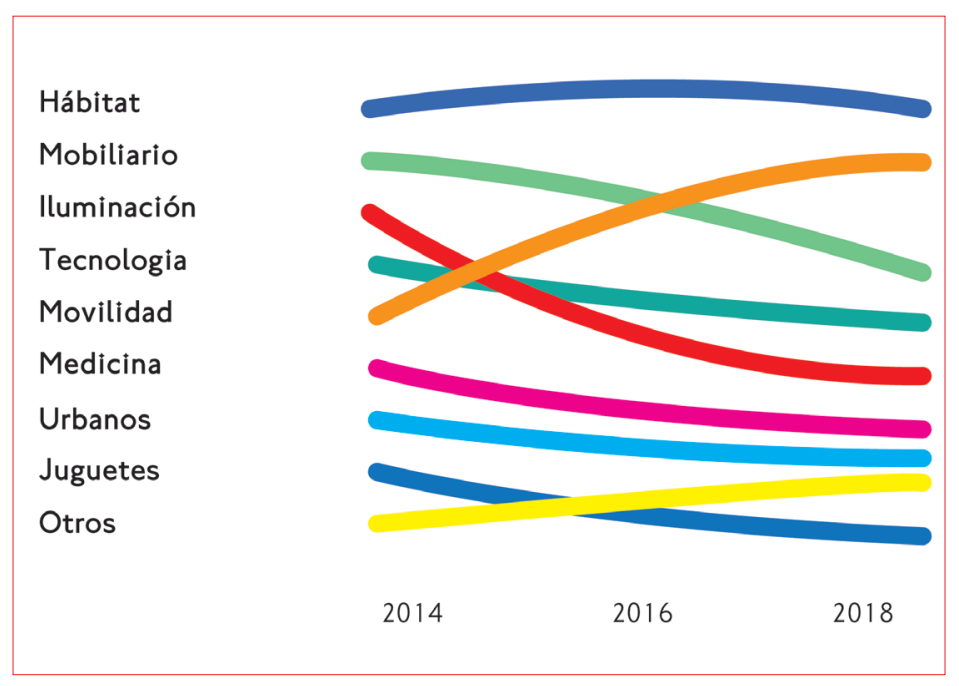

Figura 2. Ámbitos de los proyectos.

bién deberemos considerar los proyectos participativos resultados de procesos de cocreación en grupo con colectivos específicos. Liz Sanders (2008) define la cocreación como una herramienta que puede hacer que personas de diferentes disciplinas puedan comunicarse mejor y puedan colaborar para la realización de proyectos en empresas, comunidades y colectivos. Spencer Kagan (2001) define que una estructura de trabajo cooperativo necesita apoyarse en cuatro principios básicos: la participación equitativa o igualitaria, la interacción simultánea, la interdependencia positiva y la responsabilidad individual.

Siguiendo con nuestro estudio y tomando como referencia la figura 1 y la presentación por sexos, también hemos observado un aumento del sector femenino en la participación, con unos resultados muy similares de participación para ambos sexos.

\section{Nacionalidad}

La nacionalidad de los participantes es mayoritariamente española, pero hay un crecimiento en la participación de estudiantes de otras nacionalidades, provienen de países como Italia (5), Colombia (3), México (3), Suiza, Polonia, México, Islandia, Japón, Ecuador, Arabia Saudí, Kazakh, Venezuela y Argentina.

\section{Estudios y centros}

Solamente pueden presentarse al concurso alumnos que han cursado el $1^{\circ}$ nivel de CFGS, $2^{\circ}$ curso de Grado y $3^{\circ}$ curso (Máster) ya que son los que en su plan de estudios realizan el proyecto de fin de carrera. Hemos observado que los estudiantes de Grado y específicamente del Grado en Diseño y de los Estudios Artísticos Superiores en Diseño son los que más han participado. Las escuelas que más proyectos presentan en todas las tres ediciones del concurso son la Escuela "Elisava" y la Escuela "Llotja", en todas las ediciones la participación de proyectos fue mayoritariamente de centros de Catalunya. También participaron las universidades de Valencia, Castellón, Sevilla, Zaragoza y "Kingston University". Las Escuelas Superiores de Diseño de las Islas Baleares, Valencia, Alcoy, Asturias y La Rioja. En la edición de 2018 hubo también participación de mayor afluencia de centros extranjeros como "Pratt Institute" y el "Royal College of Art".

\section{Ámbito de los proyectos}

En la recogida de datos de los ámbitos del proyecto, hemos categorizado los diferentes proyectos presentados en función de las especialidades profesionales de los estudios de diseño.

Los resultados obtenidos muestran que desde el 2014 al 2018 la mayoría de los proyectos presentados (20\%) pertenecen al "ámbito del hábitat”. Por otro lado, entre 2014 y 2018, hay un aumento de productos relacionados con otras especialidades como son la moda (+8\%) y la movilidad $(+5 \%)$.

Los ámbitos más tradicionales como la iluminación (-9\%), el mobiliario (-4\%) y los productos para el espacio urbano (-2\%) reducen el número de proyectos presentados en la última edición. En todas las ediciones destaca la baja representación de proyectos de productos para el sector médico $(-8 \%)$, productos tecnológicos $(-6 \%)$ y juguetes $(-2 \%)$.

Es interesante visualizar en la figura 2, cómo han evolucionado durante los últimos años, puesto que el Diseño tiene muchos campos de aplicación y plantea diferentes oportunidades más allá de los ámbitos tradicionales.

\section{Tipologías}

A partir de las intenciones o finalidades en las que se han desarrollado las temáticas de los proyectos, hemos clasificado y agrupado, los diferentes proyectos de acuerdo a las siguientes tipologías: comercial, experimental, social, especulativo y servicios.

El tipo de proyecto comercial englobaría los diseños clásicos donde se propone un producto para un cliente concreto con una especial consideración del mercado y público al que va dirigido. También incluiría el diseño de un producto me- 
diante un sistema de auto producción con mecanismos de autofinanciación. Según las aportaciones de Alex Milton (2013) y John Lindbeck (1995). Como ejemplo de trabajo premiado mostramos "Plow 2.0", que fue Medalla Oro 2016, un nuevo sistema de pala de rescate, que rompe con la tradicional pala extensible de un solo mango ( monomango).

El tipo de proyecto experimental, es un tipo de proyecto de diseño que tiene como objetivo principal la exploración, la experimentación y el descubrimiento a partir de materiales, tecnologías, procesos de fabricación, conceptos o cuestiones estéticas con un alto grado de innovación. Aunque finalmente se concretará en algún tipo de producto su prioridad y finalidad no son los aspectos comerciales. Según las aportaciones de Lidewij Edelkoort (2012) o Ashley Hall (2011). Como ejemplo presentamos "Without", "Medalla Adi Bronce" 2018, experimentación a través de complementar el diseño de producto con técnicas que se utilizan en el diseño de moda, pasar del corte en $2 d$ a un producto en $3 d$ de la forma más creativa.

El tipo de proyecto social, sería un tipo de proyecto de diseño con vocación de servicio dirigido a satisfacer las necesidades de aquellos colectivos de personas ignoradas por la industria por que pertenecen a colectivos minoritarios o muy reducidos y por tanto tienen poca capacidad adquisitiva. Habitualmente implican a las ONG, agencias gubernamentales $u$ organismos internacionales, dado su escaso rendimiento desde el punto de vista económico, no así desde un punto de vista ético y de responsabilidad moral para con los más desfavorecidos de la sociedad, según lo aportan los autores Nynke Tromp (2011), Raquel Pelta (2010) y Ezio Manzini (2015). Como ejemplo de este ámbito hemos elegido "Aux.!" mención "Adi 2018", sistema de auto fabricación de un elemento auxiliar de flotación eficaz pensado para los niños refugiados.

El tipo de proyecto especulativo, sería un tipo de proyecto de diseño donde se proponen productos que se convierten en herramientas para pensar y que pueden plantear tanto cuestiones psicológicas, políticas o sociales, como escenarios futuros $\mathrm{y} / \mathrm{o}$ imaginarios que generen un espacio de debate. Considerando las visiones de Dunne y Raby (2013) no tienen una vocación comercial ni se ven en el mercado sino que se muestran en exposiciones, libros, revistas, webs... Como ejemplo destacamos "Buzz", selección "Adi 2018”, el proyecto utiliza el placer para transgredir contra los tabúes y los códigos que dictaminan nuestros cuerpos en el espacio público.

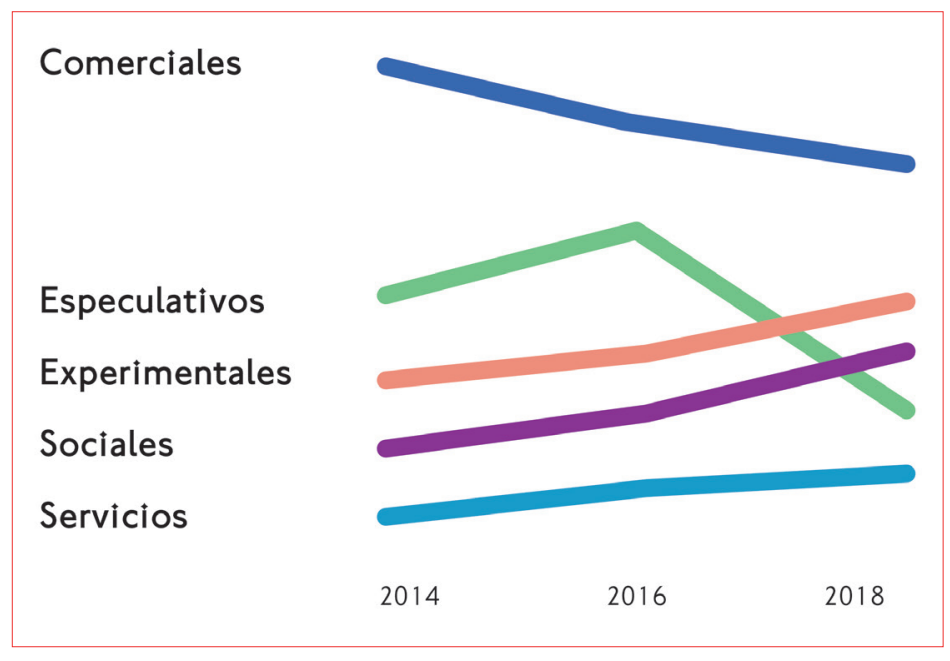

Figura 3. Tipos de proyectos

El tipo de proyecto servicios, es un tipo de proyecto donde no tiene que desarrollarse un producto físico industrializado, sino que puede ser una idea de un nuevo servicio, que puede manifestarse a través de tangibles: como productos, una aplicación web o una aplicación móvil. Teniendo en cuentas las aportaciones de Xènia Viladàs (2010) y Lara Penin (2015). Como ejemplo el "Recuerdo de Canillo", no seleccionado, unión de los puntos de interés cultural a través de un servicio, aplicando economía circular y potenciando el turismo sostenible.

El análisis de los datos, muestra que pese al predominio de la tipología de proyectos comerciales, se observa una clara tendencia a su disminución (-25\%) entre 2014 y 2018.

Destacamos la oscilación y el crecimiento de una edición de proyectos Especulativos, que puede ser debido a la participación de estudiantes del Postgrado en Concepto de Producto, de "Elisava".

Hay un aumento de proyectos de tipología Experimental $(+14 \%)$ y Social $(+12 \%)$, sin embargo los proyectos de tipo Servicios (2\%) no son suficientemente representativos.

\section{Página web personal}

Uno de los aspectos interesantes que hemos observado y queremos destacar, es el aumento por encima del doble de los registros que aportaron una página Web personal, utilizando un dominio propio o una plataforma. En la edición de 2014, el $37 \%$ hizo pública su página Web personal, de ellos la mayoría utilizaron su web personal con URL, el 

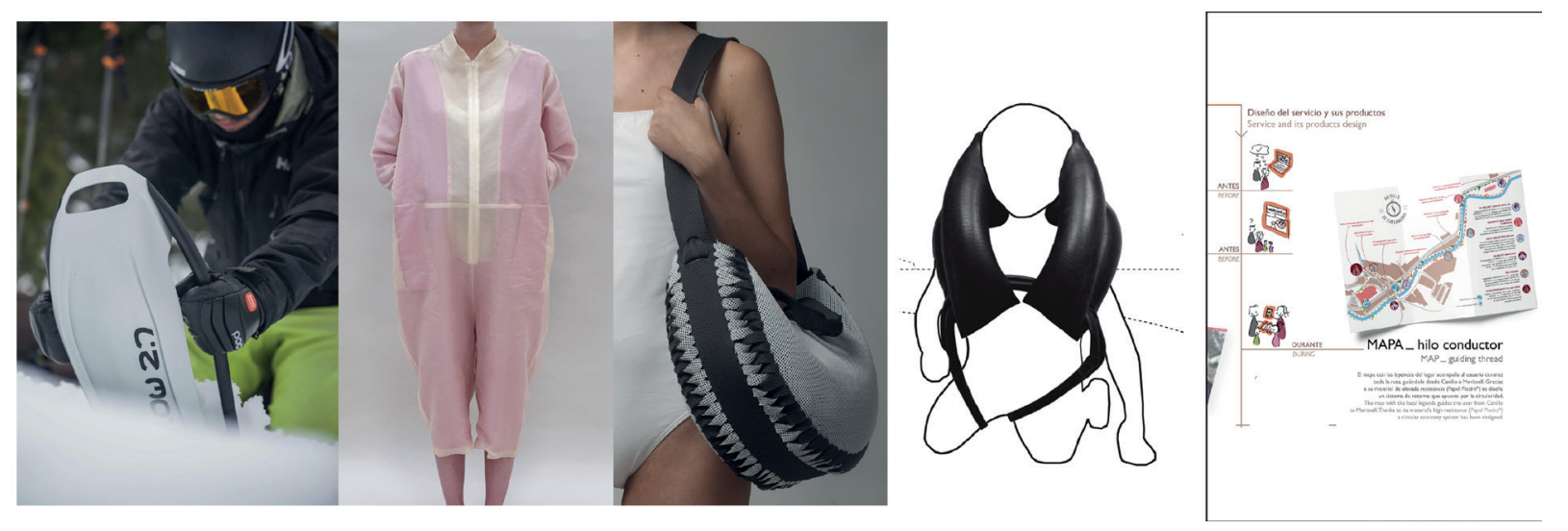

Figura 4. Ejemplos de proyectos (1:”Plow 2.0”, 2:“Without, 3:”Aux!”, 4:“Buzz”, 5:“Recuerdo de Canillo”)

resto de participantes uso su perfil con "Behance" (20\%), "Wixsite" (10\%), "Cargocollective" (5\%). En la segunda edición del 2016 subió un poco más y fue el $43 \%$ los que lo hicieron, mientras que en la edición de 2018 sí que la mayoría de los participantes, un $73 \%$ ya se registró con una página Web personal. De estos, un $44 \%$ aportaron su web personal con URL registrada y el resto presentó su perfil con "Behance" (20\%), "Wixsite" (13\%), "Cargocollective" (9\%), "Instagram" (5\%), "Carbonmade" y en un solo caso con "Wordpress". Es evidente que tanto en los centros educativos como en los propios perfiles de los estudiantes hay un aumento en el uso de estos recursos multimedia.

\section{Materiales audiovisuales complementarios}

Teniendo en cuenta el uso de materiales audiovisuales complementarios en la presentación de los trabajos, destacamos que en las últimas ediciones muchos proyectos estaban enlazados en un espacio propio de la Universidad o Escuela, esto también muestra que las propias escuelas y universidades están creando plataformas para publicar los proyectos finales de sus estudiantes.

Mientras que en 2014 apenas se colgaron recursos complementarios como son el vídeo del proyecto o la página Web del Proyecto. En 2016, sólo el $41 \%$ tenía Web personal del proyecto. No todos los proyectos tenían video, 61 proyectos no presentaron vídeo (58\%) y 44 sí tenían vídeo (42\%).

En 2018, en general (62\%) tenían una Web específica del proyecto. Este año hubo un número significativo de proyectos que también presentaron un vídeo (60\%). Es interesante observar el aumento de estos recursos registrados, en generaciones que cada vez son más digitales.

\section{Conclusiones}

Después de elaborar un análisis de los resultados de las dos unidades temáticas referentes a las características de los estudiantes y de los proyectos presentados al concurso Medallas ADI, partiendo de los diferentes indicadores estudiados, nos hemos planteado las siguientes conclusiones.

Se han producido variaciones entre el número de inscritos al concurso en las diferentes convocatorias, el número es suficientemente indicativo para la muestra (261 participantes) pero si hay un desequilibrio entre las diferentes ediciones. Se dan las circunstancias que la participación de los estudiantes no es obligatoria y puede oscilar en función de sus intereses y la comunicación de la convocatoria.

Según la variable del género de los participantes, en todas las ediciones están equilibrados el número de hombres y el de mujeres, mostrándose un aumento de mujeres en la última convocatoria.

Destacar la representación significativa de algunas escuelas que permiten hacer el trabajo final de estudios en grupo.

En los títulos de los Proyectos finales, el recurso más utilizado es inventarse una palabra y hay una tendencia alcista en escribir el título en inglés, aunque la mayoría de estudiantes son de nacionalidad española.

El ámbito de los proyectos finales más presentado sigue siendo el producto para el hábitat, han aumentado Proyectos de complementos, bolsas o piezas de moda, proyectos relacionados con "food design" y con el diseño para la movilidad. Ha habido una disminución de proyectos de iluminación y mobiliario. Destacar que en los ámbitos en los que hay menos representación, son en 
51

Guillem Ferran Masip
ESS

grafica: casi todos los casos, los ganadores del Concurso: proyectos que trabajan en mejorar aspectos para usuarios especiales (-2\%) y también los proyectos que se centran en productos para niños o que aportan o contribuyen a mejorar aspectos relacionados con el deporte.

Referente al tipo de proyectos, siguen teniendo más importancia aquellos con finalidad comercial, se constata un cierto crecimiento de proyectos experimentales y sociales versus a proyectos más especulativos, aun así, las aportaciones más representativas siguen siendo proyectos de diseño de "interfícies" y proyectos con una fuerte sensibilidad en el "ecodiseño".

La realización de este estudio comparativo nos ha permitido observar que las temáticas se repiten de un año tras otro, que hay poca apertura a nuevos conceptos, que estas limitaciones deberían interpelarnos como docentes y motivarnos a reflexionar sobre si estamos facilitando el espíritu innovador y creativo a nuestro alumnado. Es importante educar en el espíritu crítico para observar las deficiencias, las necesidades no cubiertas y para indagar y facilitar herramientas que nos lleven a plantear ayudas delante de deficiencias de tipo social, servicios para las personas mayores y más vulnerables, así como también la búsqueda para mejorar aspectos de nuevos materiales que nos lleven a un mundo más práctico, eficaz y sostenible.

En la última edición han crecido las propuestas de proyectos finales que apuestan por la innovación de nuevos materiales y nuevos procesos de producción que abren caminos para la construcción de nuevos objetos.

El aumento de estudiantes con Página Web con URL personal (más del 30\%) en estas últimas cinco generaciones, hace evidente la concienciación entre el alumnado de la importancia de esta herramienta indispensable cuando acaban sus estudios. También destacar como han aumentado el uso de plataformas usadas como "Behance", "Wixsite", "Cargocollective" o el propio "Instagram" como web personal.

También han aumentado los Proyectos finales que van acompañados de un vídeo (más del $20 \%$ ) siendo esta una herramienta indispensable en la presentación del proyecto final de estudios.

Los premios y concursos son una buena forma de comparar resultados entre estudiantes de diferentes centros. Los Premios Medalla ADI son una buena plataforma para investigar sobre cómo evolucionan los resultados de los que reciben diferentes formaciones de diseño industrial o de producto en las escuelas de este país. 


\section{Referencias bibliográficas}

Adi Fad (2014). Catálogo premios Delta 2014.

Adi Fad (2016). Catálogo premios Delta 2016.

Adi Fad (2018). Adi Book 2018. Catálogo de los premios Delta 2018.

AdiFad (2014) Base de datos de los proyectos registrados en 2014. Adi Fad: Barcelona (Descargado a través de la documentación ubicada en el ordenador).

Coller, X. "Estudio de casos." Colección cuadernos metodológicos, $\mathrm{n}^{\circ} 30$. CIS. Centro de investigaciones sociológicas: Madrid.

DUNNE, A.; RABY, F. (2013) Speculative everything design, fiction, and social dreaming. MIT.

Edelkoort, L.; Antonelli, P. (2012) The pop-up generation: design between dimensions. BIS Publishers.

Escoda, N.; Aliaga, L. (2018) Catálogo interno de registros de los premios Delta. Adi Fad: Barcelona (Descargado a través de la documentación entregada por Typeform).

HALL, A. (2011) Experimental design: design experimentation. Design issues. Volum 27. $\mathrm{n}^{\circ} 2$ pàg.17-26.

KAGAN, S. (2001) "Kagan structures and learning together. What is the difference?". Kagan Online Magazine. (http://www.kaganonline.com/ KaganClub/ FreeArticles.html)
LINDBECK, J. (1995) The design process. Product design and manufacture. Prentice Hall,

ManzinI, E. (2015) Design, when everybody designs. MIT.

Milton, A. ; Rodgers, P. (2013) Metodos de investigacion para el diseño de producto. Blume.

Pelta, R. (2010). Diseñar para cambiar. Étapes. $\mathrm{n}^{\circ} 10$.

PenIN, L. (2015) An introduction to service design: designing the invisible.

Tromp, N.; Hekkert, P., Verbeek, P.P. (2011) Design for socially responsible behavior: a classification of influence based on intended user experience. Design Issues. MIT.

Sanders, E; Stappers, P.J. (2008) Co-creation and the new landscapes of design. Co-Design, 4:1, 5-18,

Sans, T.; Calvet, P. (2016) Catálogo interno de registros de los premios Delta. Medallas. Adi Fad: Barcelona (Descargado a través de la documentación entregada por Typeform).

VAN MANEN, M. (1977) Linking ways of knowing with ways of being practical. Curriculum Inquiry, 6 , 205-228.

VILADAS, X. (2011) Design at your service: how to improve your business with the help of a designer. Index Book. 